Article

\title{
Photoactive Nanomaterials Inspired by Nature: LTL Zeolite Doped with Laser Dyes as Artificial Light Harvesting Systems
}

\author{
Leire Gartzia-Rivero, Jorge Bañuelos * and Iñigo López-Arbeloa \\ Molecular Spectroscopy Laboratory, Department of Physical Chemistry, University of the Basque Country \\ UPV/EHU, 48080 Bilbao, Spain; leire.gartzia@ehu.es (L.G.-R.); inigo.lopezarbeloa@ehu.es (I.L.-A.) \\ * Correspondence: jorge.banuelos@ehu.es; Tel.: +34-94-601-2711
}

Academic Editor: Francisco del Monte

Received: 30 March 2017; Accepted: 28 April 2017; Published: 4 May 2017

\begin{abstract}
The herein reported work describes the development of hierarchically-organized fluorescent nanomaterials inspired by plant antenna systems. These hybrid materials are based on nanostructured zeolitic materials (LTL zeolite) doped with laser dyes, which implies a synergism between organic and inorganic moieties. The non-interconnected channeled structure and pore dimensions (7.1 $\mathrm{A})$ of the inorganic host are ideal to order and align the allocated fluorophores inside, inferring also high thermal and chemical stability. These artificial antennae harvest a broad range of chromatic radiation and convert it into predominant red-edge or alternatively white-light emission, just choosing the right dye combination and concentration ratio to modulate the efficiency of the ongoing energy transfer hops. A further degree of organization can be achieved by functionalizing the channel entrances of LTL zeolite with specific tailor-made (stopcock) molecules via a covalent linkage. These molecules plug the channels to avoid the leakage of the guest molecules absorbed inside, as well as connect the inner space of the zeolite with the outside thanks to energy transfer processes, making the coupling of the material with external devices easier.
\end{abstract}

Keywords: hybrid nanomaterials; luminescent antennae; energy transfer; LTL zeolite; laser dyes; stopcock molecule

\section{Introduction}

The development of photoactive nanomaterials capable of mimicking the functions and mechanisms present in nature has become a major challenge for scientists of different fields [1-3]. These systems show an incredibly high efficiency and perfection, which tempted the scientific community to simulate them by designing artificial systems that can further guarantee environmentally-friendly technological progress. Photosynthetic organisms present in plants, for instance, appear as the most sophisticated solar energy storage systems in nature due to their unique ability to harvest solar radiation and transform it into chemical energy. The light is absorbed by antenna systems composed of a few hundred chlorophyll molecules embedded in a protein environment, which keeps the photoactive moieties well arranged and with the proper orientation to transfer the excitation energy efficiently to a specific reaction center [4,5]. Nowadays, many efforts are being made to fully understand the complex process of photosynthesis, since it involves many other fundamental processes necessary for the proper performance of the complete system [6]. In this regard and taking inspiration from plant antenna systems, many imaginative attempts have been tested to build artificial luminescent antennae, among them self-assembled nanofibers [7], dendrimers [8], luminescent metal complexes [9], DNA nano-scaffolds [10], dyes embedded in polymer matrices [11] and host-guest hybrid systems [12]. 
One of the main requirements for the proper functioning of an artificial antenna system is the ability to harvest and transport the light to an acceptor moiety with the desired energy (Figure 1). In this sense, the excitation energy transfer (EET) appears as the key factor ruling the effectiveness of the process. Therefore, a thorough understanding of the parameters controlling this phenomenon is necessary, such as the ratio of donors and acceptors, interchromophoric distances, relative orientations and spectral overlap [13]. The accurate control of all of these variables leads to the development of competitive and efficient antenna systems with potential applications in photonics, optical coding, biosensing, catalysis, photosynthetic, logic devices and theranostics, among others [14-16].

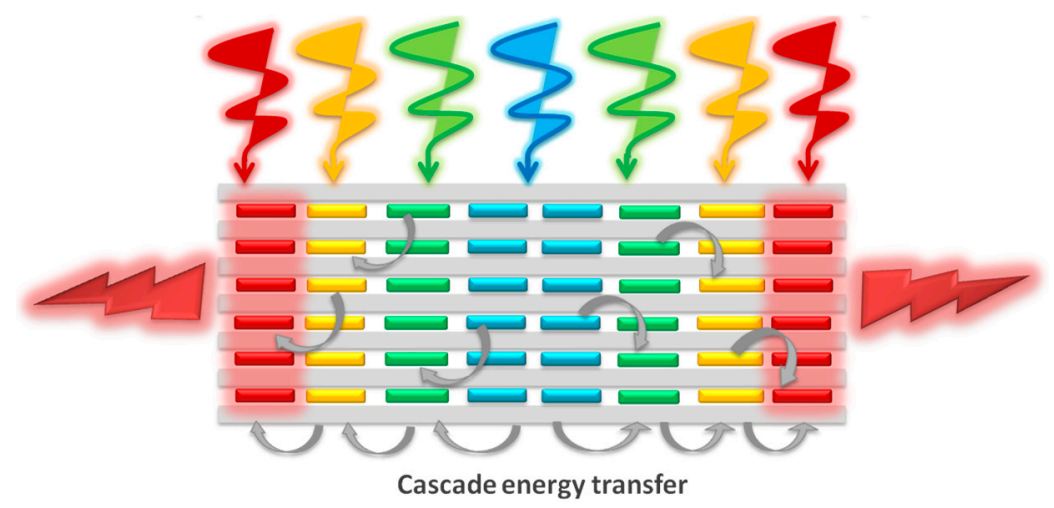

Figure 1. Schematic overview of an artificial luminescent antenna.

As an attempt to develop competitive artificial antenna systems, the design of photoactive organic-inorganic hybrid materials shows promising possibilities to mimic the perfection and effectiveness of the antenna systems present in plants. In the herein presented work, the protein matrix of the natural systems has been replaced by a zeolite host (Linde Type L, LTL) of nanometric dimensions, which protects the dyes and provides a significant degree of arrangement $[17,18]$. Moreover, with respect to the photoactive moiety, responsible for interacting with the light, the chlorophyll molecules have been replaced by fluorescent molecules. Indeed, and thanks to the advances in organic chemistry, nowadays, there is a wide chart of commercially-available laser dye families, which cover the ultraviolet, visible and near-infrared regions of the electromagnetic spectrum [19].

The incorporation of an organic photoactive guest into a nanostructured solid matrix is an active area of research, reflected by the high number of related publications [20-22]. The strategy of encapsulation can avoid the formation of aggregates, improve the chemical and thermal stability of the dye and force it to adopt a preferred orientation, creating highly organized photoactive hybrid materials. Furthermore, the synergism between the organic and inorganic components could be used to monitor the physicochemical properties of the solid framework taking advantage of the sensing capabilities of the fluorophore [23].

Among the natural and synthetic zeolites discovered up to date, the LTL zeolite appears as a suitable candidate to allocate fluorescent dyes. Its unidimensional channel system running along the main axis of the crystal and a cylindrical pore size of a diameter of around $7.1 \AA$ A provide a constrained framework in which the molecules fit like a glove, thereby rendering well-organized materials (Figure 2). Therefore, this kind of molecular sieve is an excellent candidate for shape-selective hosting guests (such as ions, metals or organic molecules). The LTL zeolite crystals are usually achieved by hydrothermal synthesis (even assisted by microwave heating) with a tunable size and morphology [24-28]. 


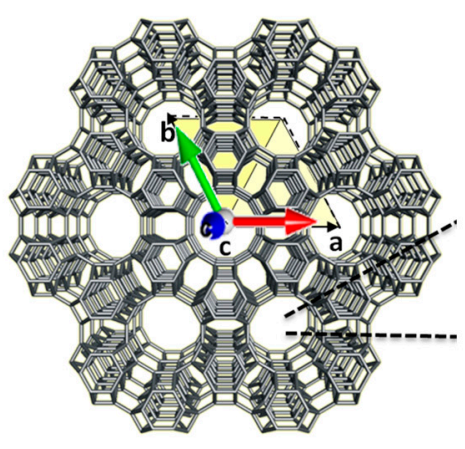

(a)

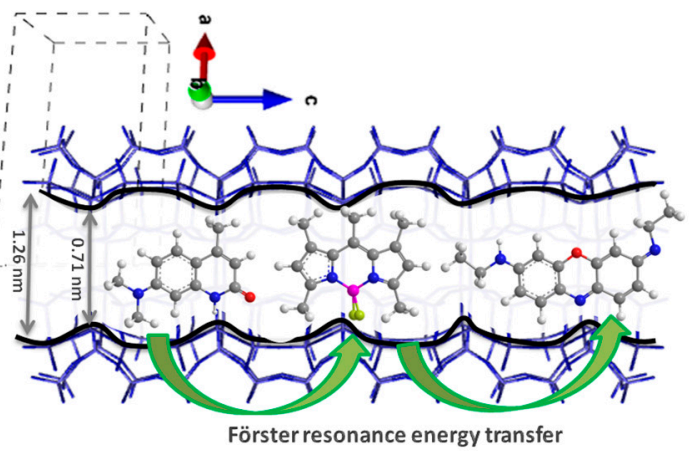

(b)

Figure 2. Top view of the LTL zeolite framework, illustrating the hexagonal structure (a); and the side view of the channel with a van der Waals opening of $0.71 \mathrm{~nm}$ at the smallest and $1.26 \mathrm{~nm}$ at the widest place (b), suitable for allocating luminescent fluorophores.

In this work, we aim to build hybrid photoactive materials as artificial antennae by the sequential insertion of luminescent dyes, working in the blue (oxazole and carbostyril), green-yellow (BODIPYs) and red-edges (oxazines and modified BODIPY) of the electromagnetic spectrum, in the unidimensional channels of LTL zeolite synthesized by microwave heating with a tunable size and morphology. This strategy allows harvesting light over a broad spectral range and transferring efficiently via the Förster resonance energy transfer (FRET) mechanism in a cascade-like unidimensional process from the center to the crystal ends (Figures 1 and 2). The encapsulation of the dyes ensures short donor-acceptor distances to switch on such FRET. An accurate control of the energy transfer efficiency (by means of the donor-acceptor spectral overlap and ratio), as well as an adequate selection of the combined dyes (mainly the final acceptor red-emitting dye on the basis of its fluorescence efficiency) allows a fine modulation of the emission window, from predominant red emission to white-light, and opens the door to a new generation of photoactive materials capable of modulating the emission light output. Moreover, a further degree of organization is provided by the covalent linkage of a stopcock molecule working in the red-edge of the electromagnetic spectrum, which acts as the final energy acceptor and allows the connection of the material to external devices. Therefore, the resulting dye-doped LTL zeolite materials are highly versatile and potential candidates to be used as tailor-made devices for multiple applications.

\section{Results and Discussion}

\subsection{Energy Transfer across the Channels of Dye-Doped LTL Zeolite}

The first step to develop antenna systems and white-light-emitting devices is to select dyes working in different regions of the electromagnetic spectrum and susceptible -of promoting efficient intermolecular energy-transfer processes within the LTL zeolite channels [29-31]. The energy transfer takes place via dipole-dipole coupling, better known as the through-space Förster mechanism, where the main requirement is the proper spectral overlap between the emission spectrum of the donor and the absorption spectrum of the acceptor (see Figures S1-S3 in the Supporting Information showing the spectral overlaps of the chosen dye combinations) [13]. Moreover the selected dyes should be small enough to diffuse along the channels, but large enough to avoid the formation of aggregates (mainly the non-fluorescent H-type). To ensure broadband absorption along the whole UV-Vis spectral region, three different dyes were successively allocated in the LTL zeolite pores. As energy donors, oxazole (Dmpopop) and carbostyril (C165) dyes have been considered in the UV-blue region, whereas in the green-yellow part, BODIPYs (PM546 and PM567) have been selected as first acceptors and ulterior energy donors. Lastly, as final energy acceptors, red-emitting oxazines (Ox1 and Ox4) were chosen. Table 1 summarizes the donor-acceptor dye loading and ratio, as well as key parameters 
to describe the FRET probability (such as spectral overlap or critical transfer distance) between the donor-acceptor pairs in each of the herein-tested antennae. The sequential insertion of the fluorophores leads to a hierarchically-organized material in which the UV-blue donors are placed in the center of the crystals, flanked by the green-yellow dyes, and with the red acceptors localized at the edges of the LTL zeolite crystal (Figure 3). In fact, the dimension and constrained environment of the channel framework not only avoid the dyes sliding one over each other, but also provide a short donor-acceptor distance, which improves the FRET efficiency. Therefore, by properly choosing the dyes to be allocated and playing with the spectral overlap and donor/acceptor ratios of the combined dyes, the FRET efficiency can be finely tuned, and hence, the output emission light of the photoactive material may be modulated accordingly.

Table 1. Meaningful data about dye loadings, spectral band positions and the ongoing FRET between the dyes occluded or anchored into the disc-shaped LTL zeolite: dye loadings, donor-acceptor ratio, spectral overlap $(\mathrm{J})$, absorption $\left(\lambda_{\mathrm{ab}}\right)$ and fluorescence $\left(\lambda_{\mathrm{fl}}\right)$ peak wavelengths. C165, carbostyril; Ox, oxazine.

\begin{tabular}{|c|c|c|c|c|c|c|c|}
\hline & Dyes & $\begin{array}{c}\text { Dye } \\
\text { Loading a (\%) }\end{array}$ & $\begin{array}{c}\text { Dye } \\
\text { Ratio }\end{array}$ & $\left(10^{-13} \mathrm{M}^{-1} \mathrm{~cm}^{3}\right)$ & $\begin{array}{l}\mathbf{R}_{0}{ }^{c} \\
(\AA)\end{array}$ & $\begin{array}{c}\lambda_{\mathrm{ab}} \\
(\mathrm{nm})\end{array}$ & $\begin{array}{c}\lambda_{\mathrm{fl}} \\
(\mathrm{nm})\end{array}$ \\
\hline \multirow{3}{*}{ } & Dmpopop:PM567: Ox1 & 10:10:10 & $1: 1: 1$ & $\begin{array}{c}\text { D1-D2 = } 1.0 \\
\text { D2-A }=1.8\end{array}$ & $\begin{array}{l}55.0 \\
65.8\end{array}$ & $\begin{array}{l}364 \\
518 \\
646\end{array}$ & $\begin{array}{l}429 \\
539 \\
669\end{array}$ \\
\hline & \multirow[t]{2}{*}{ C165:PM546: Ox4 } & 10:10:10 & $1: 1: 1$ & $\begin{array}{c}\text { D1-D2 }=1.1 \\
\text { D2-A }=2.0\end{array}$ & $\begin{array}{l}59.1 \\
67.0\end{array}$ & $\begin{array}{l}360 \\
495 \\
614\end{array}$ & $\begin{array}{l}424 \\
509 \\
638\end{array}$ \\
\hline & & 10:10:2 & $1: 1: 0.2$ & $\begin{array}{c}\text { D1-D2 }=1.1 \\
\text { D2-A }=2.0\end{array}$ & $\begin{array}{l}59.1 \\
67.0\end{array}$ & $\begin{array}{l}360 \\
495 \\
614\end{array}$ & $\begin{array}{l}424 \\
509 \\
638\end{array}$ \\
\hline$\underset{\pi}{\dddot{\theta}}$ & Dmpopop: Stopcock & $10:^{*}$ & 1:0.05 & 0.06 & 34.4 & $\begin{array}{l}364 \\
558\end{array}$ & $\begin{array}{l}436 \\
599\end{array}$ \\
\hline
\end{tabular}

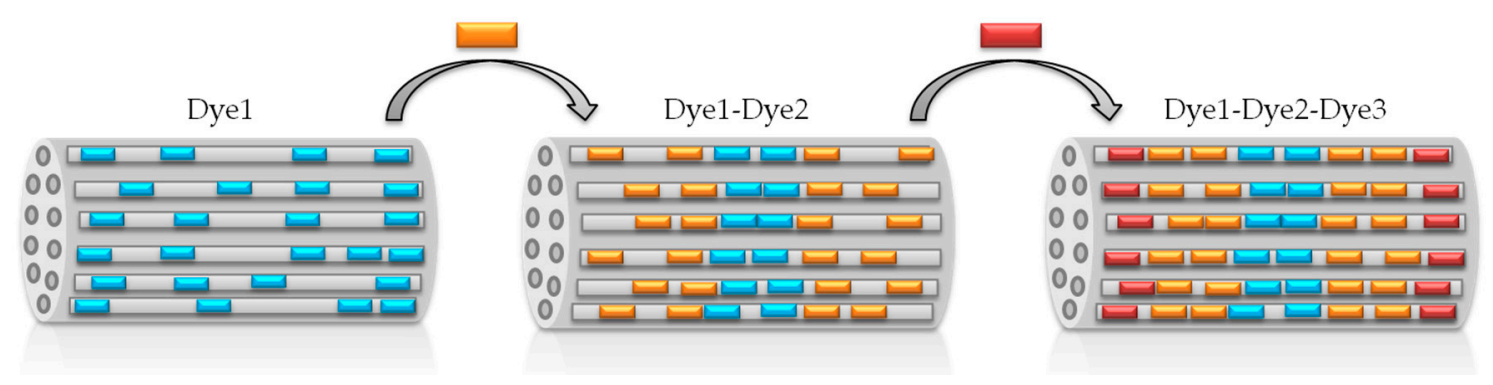

Figure 3. Sketch of the sequential insertion of the fluorophores into the LTL zeolite channels.

\subsubsection{Dmpopop, PM567,Ox1-Doped LTL Zeolite}

The herein presented antenna system is composed of the sequential insertion of Dmpopop-PM567-Ox1 dyes into the zeolitic channels of disc-shaped crystals (see Section 3.2 for details and Table 1) [32]; in particular, following the aforementioned sequential order, first the donor Dmpopop, then the BODIPY and, finally, the oxazine. These dyes kept their identity inside the channels, in spite of the imposed proximity by the constrained framework, ruling out intermolecular aggregation and thereby displaying their characteristic absorption bands spread along the UV-Vis region [32]. As a result, this antenna entails an effective light harvesting over a broad spectral region 
(Table 1). After selective excitation at the UV region, where just the first energy donor (Dmpopop) absorbs, its own emission is recorded but followed by that from PM567 and the last energy acceptor Ox1 emission band (Figure 4). Therefore, this hybrid material undergoes FRET (see spectral overlap in Figure S1, which enables through-space FRET) as red emission is attained upon excitation far away from the long-wavelength absorption of oxazines, but partial, since the emission from the donors is not completely quenched and the corresponding fluorescence of each chromophore is simultaneously detected. Nonetheless, the sum of all of these emissions covering the whole visible spectrum leads to white-light emission (Figure 4) in which the energy output can be finely tuned over a broad region just using suitable filters $[33,34]$.

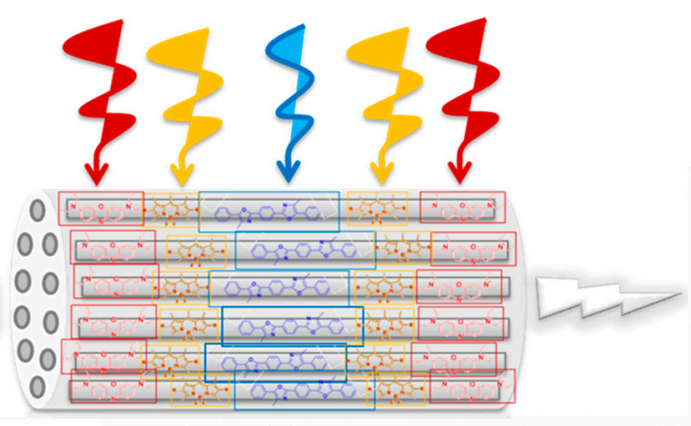

(a)

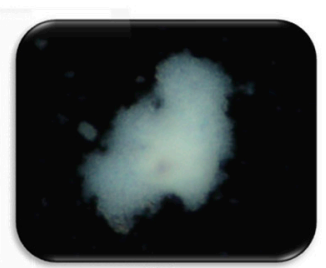

(b)

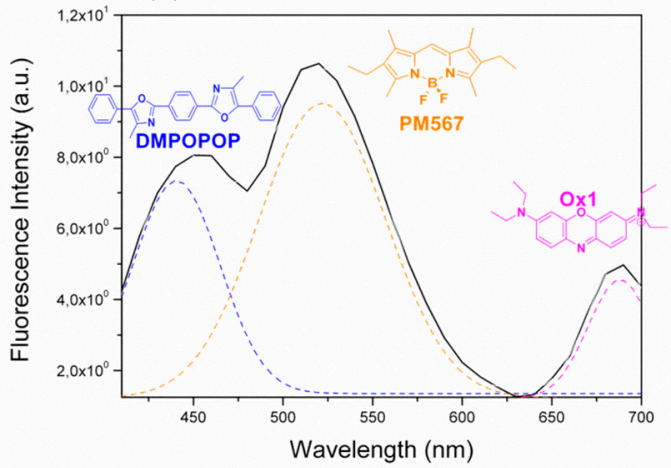

(c)

Figure 4. (a) Schematic representation of the antenna material prepared by the sequential insertion of three different dyes into disc-shaped LTL zeolite channels; (b) Fluorescence image of an agglomerate of the corresponding doped crystals under 350/50-nm excitation and recording the emission with a 400-nm long-pass filter; (c) Fluorescence spectrum recorded upon selective excitation of the first donor at $375 \mathrm{~nm}$ (dashed lines show the corresponding emission of each dye separately).

\subsubsection{C165,PM546,Ox4-Doped LTL Zeolite}

In order to transform most of the harvested light into red emission, we decided to improve the energy transfer efficiency by choosing dyes with stronger spectral overlap (see Figure S2 in the Supporting Information and Table 1) and, hence, boost the FRET pathway. For that purpose, we replaced the oxazole by a carbostyril (C165) as the blue emitting energy donor, PM567 by another BODIPY (PM546) in the green-yellow region and Ox1 by another oxazine (Ox4) with improved fluorescence capability as the final acceptor. Indeed, as a consequence of the higher spectral overlap, the critical transfer distance (also known as the Förster distance [13]) increases for each donor-acceptor pair of this antenna with regard to the aforementioned one in Section 2.1.1, anticipating an improved FRET efficiency for the new designed antenna (Table 1). For a proper comparison with the above tested antenna, the rest of the parameters were not altered, which means, the same dye loadings into disc-shaped LTL zeolites $(10 \%$ of the available free adsorption sites occupied for each dye; see Section 3.5 for details), thus keeping also the same donor/acceptor ratio at 1:1:1. 
Again, the presence of each dye is verified by the detection of their characteristic bands in the corresponding spectral region spanning almost the whole UV-Vis spectrum (Table 1). After UV excitation, the emission of each chromophore is recorded as a consequence of the expected cascade-like FRET processes from the carbostyril to the neighboring BODIPY and from there to the adjacent oxazine through the unidimensional channel preferably (Figure $5 b$ ). However, the ratio between the relative fluorescence intensities of each band changes. Whereas in the previous antenna, the blue and green emission prevails, the red one being less intense, in this case, a much more prominent red-edge emission appears, by far being the most intense peak in the spectrum.

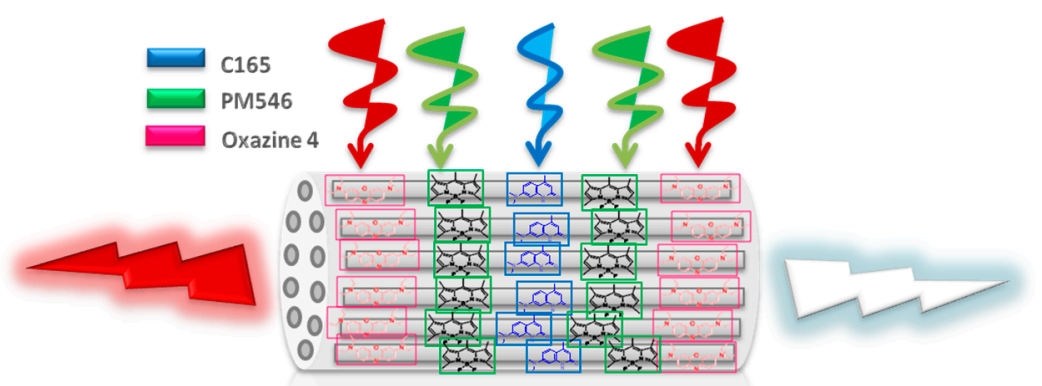

(a)

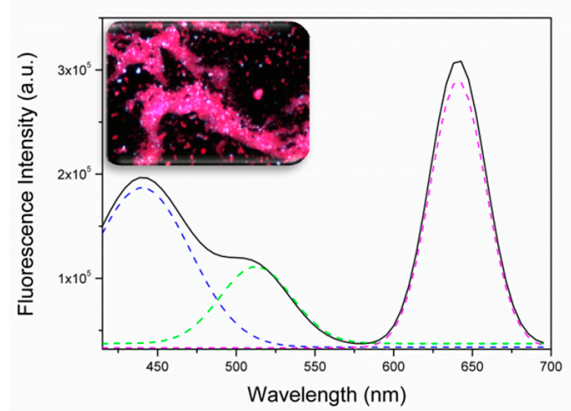

(b)

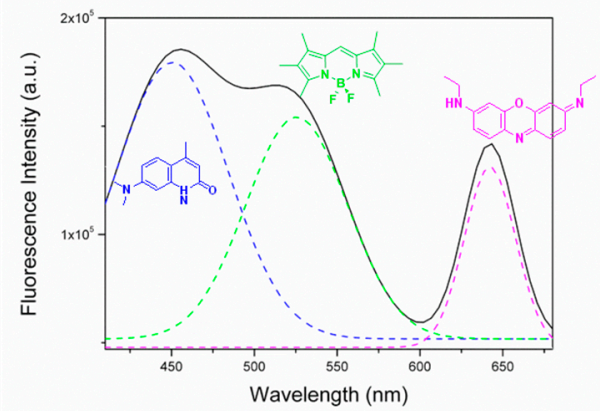

(c)

Figure 5. (a) Schematic representation of the antenna material prepared by the sequential insertion of three different dyes into disc-shaped LTL zeolite channels. Fluorescence spectrum of (b) C165(10\%)-PM546(10\%)-Ox4(10\%)- and (c) C165(10\%)-PM546(10\%)-Ox4(2\%)-doped LTL zeolites recorded upon selective excitation of the first donor at $375 \mathrm{~nm}$ (dashed lines account for the emission of each free dye). Inset in (b): fluorescence image of agglomerated dye-doped zeolite crystals under 350/50-nm excitation and recording the emission with a long-pass filter of $400 \mathrm{~nm}$.

Such evolution is a consequence not only of the ameliorated FRET efficiency, but also of the enhanced fluorescence quantum yield of the final energy acceptor Ox4 with regard to Ox1 [35]. As a result of this synergistic effect, the donor fluorescence quenching by FRET is more effective in profit of the emission from the final and brighter red-edge emitting Ox4 acceptor. Therefore, the control of the energy transfer efficiency together with the improvement of the fluorescence performance of the emitting final energy trap enables the modulation of the emission window, thus passing from the detection of emission bands with similar intensity across the visible region (white-light in Section 2.1.1) to a predominant red emission, keeping always a broadband absorption of the incoming light. Such fine modulation is achieved just by a careful selection of the encapsulated dyes.

Another alternative strategy to modulate the FRET efficiency for a given dye mixture, and hence trigger the output emission from the hybrid material, is changing the donor/acceptor ratio [21], which rules the donor-acceptor distance and, hence, modulates the FRET rate constant [13]. Accordingly, we tested such an approach in the above antenna comprising C165-PM546-Ox4 by reducing the final acceptor loading from $10 \%$ to $2 \%$ (thereby from a 1:1:1 ratio to 1:1:0.2; see Table 1). Such a decrease of the acceptor amount implies a reduction of the intensity of the red-edge emission from the oxazine 
upon selective excitation of the first donor Dmpopop (Figure 5c). As a result of this partial energy transfer three emission bands, corresponding from each dye allocated in the zeolitic pores, with similar intensity are recorded simultaneously along the whole visible region, rendering again white-light emission output (as in Section 2.1.1, where a different dye mixture was used). These spectral features are a consequence of a decrease in the energy transfer efficiency, inherent to the diminution of the number of available energy traps responsible for absorbing the excitation energy coming from the donors [36]. Therefore, the emission window can be modulated in a straightforward way from predominant red-edge to white-light emission as in Section 2.1.1, but in this case, adjusting the ratio between donors and acceptors for a given donor-acceptor combination.

\subsection{External Functionalization of LTL Zeolite}

Once having checked that the dye occlusion in the LTL zeolite pores is a suitable strategy to develop artificial antennae owing to the promoted FRET pathway, we aim to add a further degree of organization by functionalizing the channel entrances of LTL zeolite with specific tailor-made molecules (stopcock) via covalent linkage (Figure 6) [37,38]. Such molecules grafted at the pores plug the channels to avoid the leakage of the guest molecules adsorbed inside the pores and connect the inner space of the zeolite with the outside thanks to FRET processes, in which the stopcock molecule would act as the final acceptor, making the coupling of the material with other external devices easier. The selective functionalization of the channel entrances is feasible owing to the dissimilar chemical and physical properties of the base of the cylindrically-shaped LTL crystals, bearing the channel entrances, and those of the coat.

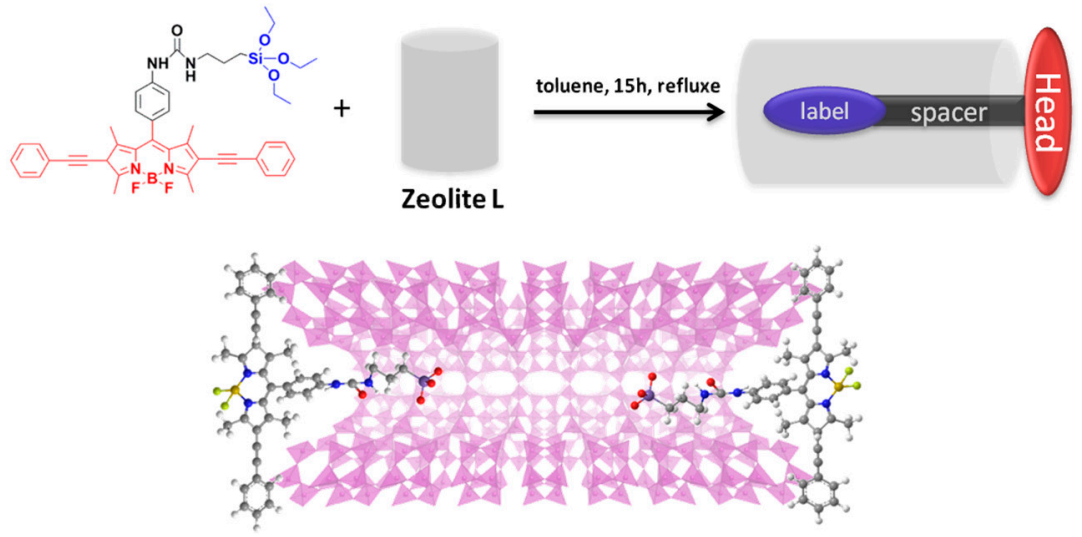

Figure 6. Molecular structure of the stopcock and the reaction principle to attach it to the LTL zeolite channel entrances. The spacer and label are located inside the channel, whereas the head is too big to enter.

A rational design of these stopcock molecules plays a key role. First it should be able to diffuse into the pores and subsequently covalently graft at the channel entrances by reaction with free silanol $(\mathrm{Si}-\mathrm{OH})$ groups, while keeping intact the photophysical properties of the chromophore. At the same time, the emission should be modulated by appropriate functionalization, so as to shift it to the red-edge of the visible to act as suitable energy acceptor of the light harvested inside the zeolite channels [39]. Keeping all of these requirements in mind, we have designed a molecule featuring a label, a spacer and a head moiety, taking as the scaffold the BODIPY dye owing to the exceptional chemical versatility of its dipyrrin core amenable to a wide assortment of synthetic reactions (Figure 6) [40,41]. Accordingly, the head consists in the tetramethylated dipyrrin bearing two acetylenephenyl arms at $\beta$-pyrrolic positions to extend the chromophoric $\pi$-system and push the spectral bands towards the red-edge. The size of this head is high enough as to avoid its diffusion into the channels and plug the entrances. The label is an organosilane moiety linked to the above head through a spacer bearing a long enough polymethylene chain at the para position of a sterically-hindered phenyl grafted at the chromophoric 
meso position (Figure 6). Such a constrained structure around the key meso position is essential to avoid any non-radiative pathway associated with the conformational freedom of the aryl and to keep the excellent fluorescence response of BODIPYs [42]. The triethoxysilane label penetrates into the channels and reacts with the zeolitic silanols after hydrolysis and polycondensation reactions, plugging the channel entrances. In fact, the fluorescence microscopy image recorded with microsized crystals (around $2 \mu \mathrm{m}$ in Figure $7 \mathrm{~b}$ ) reveals that the stopcock molecules are successfully grafted to the microsized LTL zeolite crystal, and this takes place preferentially in the channels' entrances because the density of the silanol groups is higher than in the coat. Furthermore, the photophysical properties of the stopcock are unaltered after the covalent binding, as its spectroscopic signatures remain similar to those recorded in solution (Figure 7a).

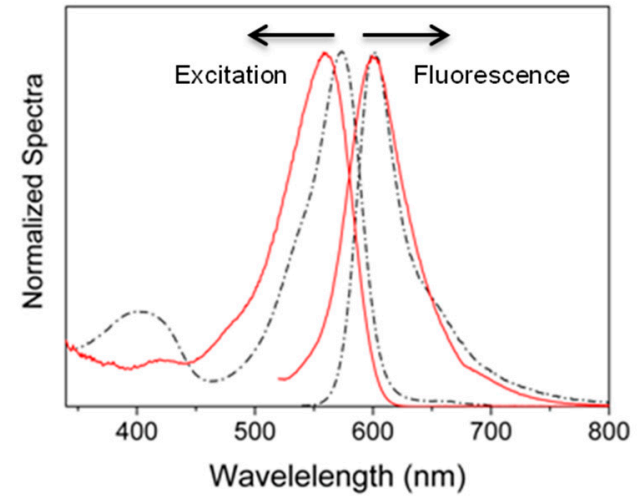

(a)

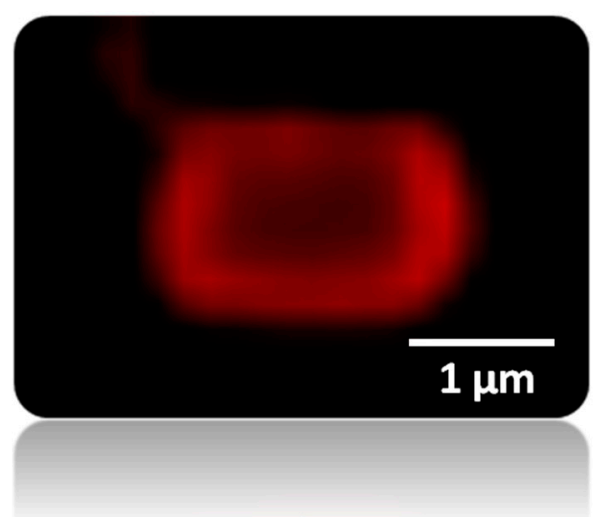

(b)

Figure 7. (a) Normalized excitation and emission spectra of the BODIPY stopcock grafted to disc-shaped LTL zeolite crystals (solid) and free in toluene (dash dotted); (b) Fluorescence microscopy image of a single microsized LTL crystal functionalized with the BODIPY stopcock.

Once having checked that the designed closure molecule is valid, we proceeded to build the antenna system by its combination with a suitable energy donor. In this sense, Dmpopop has been again chosen because it has a broad absorption band, and its emission band overlaps well with the absorption band of the stopcock (see Figure S3 in the Supporting Information) so as to undergo FRET (Table 1). Thus, the Dmpopop donor has been allocated inside the channels, and afterwards, the entrances were plugged with the silylated BODIPY. To this aim, disc-shaped LTL zeolite has been again selected owing to its morphology, characterized by a basal surface much higher than the coat (low aspect ratio, $<1$; see Section 3.2 for details), which ensures that the stopcock molecules tend to react mainly with the silanol groups of the channel entrances rather than those on the coat. Selective excitation of Dmpopop leads to a predominant red fluorescence from the stopcock at around $610 \mathrm{~nm}$ (Figure 8), supporting the ongoing FRET process from the inside to the outside of the crystal along the channel direction. Further evidence is gathered by the corresponding excitation spectrum monitored at long wavelengths, where just the stopcock emits. Indeed, two clear bands are recorded, that expected from the silylated BODIPY at lower energies and another at higher energies from the occluded Dmpopop donor. It is noteworthy that the FRET is still highly efficient, albeit the donor-acceptor distance should be longer than when both dyes are inside the channels. Likely energy migration between the donor molecules in the channels contributes to enlarging the effective distance for FRET and reaches the stopcock energy traps placed further away at the entrances.

Summing up, the coupling of an external stopcock fluorophore as the energy acceptor at the ends of the LTL zeolite channels allows a second stage of organization, in which the excitation energy is transported by donor molecules placed in the inner space of the channels and finally injected to the acceptor molecules placed at the entrances. Thus, the stopcock molecules enable the communication between the guest molecules allocated inside the pores with external materials or molecules outside 
and also prevent small molecules like $\mathrm{H}_{2} \mathrm{O}$ or $\mathrm{O}_{2}$ (fluorescence quenchers) from diffusing into the interior of the crystals.

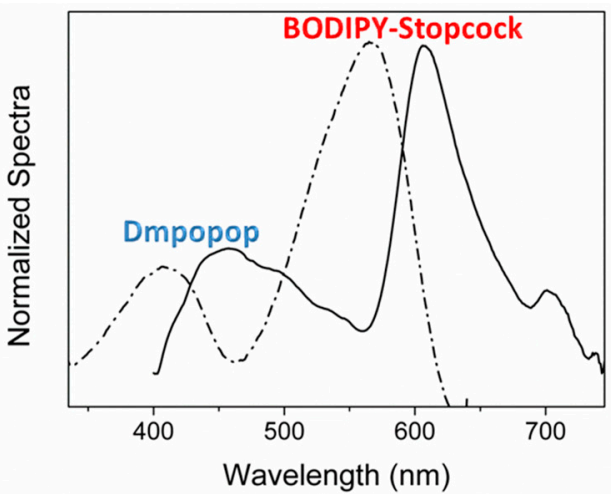

(a)

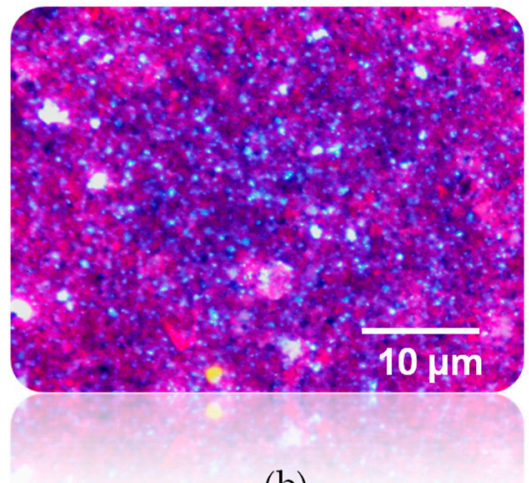

(b)

Figure 8. (a) Normalized excitation (emission measured at $660 \mathrm{~nm}$, dashed line) and emission (excited at $350 \mathrm{~nm}$, solid line) spectra of DMPOPOP enclose into the channels and BODIPY stopcock grafted in the disc-shaped LTL zeolite entrances; (b) Fluorescence microscopy image of the hybrid zeolite-dye system under 350/50-nm band-pass excitation and recording the emission with a 400-nm long-pass filter.

\section{Materials and Methods}

\subsection{Dyes}

Laser-grade BODIPY dyes, (PM546 and PM567) were purchased by Exciton, while oxazine 1 and 4, oxazole (Dmpopop) and carbostyril (C165) were purchased by Across Organics and used as received without further purification. The BODIPY-based stopcock molecule presented in Section 2.2 was synthesized by Dr. Yi Xiao from Dalian University (Dalian, China).

\subsection{LTL Zeolite Synthesis}

Zeolite crystals with a tunable size (from around $100 \mathrm{~nm}$ to $2 \mu \mathrm{m}$ ) and morphology (from barrels to discs or coins) were attained by adjusting the conditions of the hydrothermal synthesis assisted by microwave heating. Further details of the synthesis and characterization of the crystals can be found elsewhere [43]. In particular, in the herein reported work, disc-shaped zeolites (average thickness of $200 \mathrm{~nm}$ and average diameter around $1 \mu \mathrm{m}$ ) were used as hosts for the antennae, whereas barrels (length around $2 \mathrm{~mm}$ ) were used for the measurements of stopcock localization under the fluorescence microscope.

\subsection{Dye Incorporation}

Previous to dye incorporation, the zeolite was pre-treated with cesium $\left(\mathrm{Cs}^{+}\right)$cations to decrease the characteristic acidity of the zeolitic channels and adjust the $\mathrm{pH}$ to avoid the degradation or parallel acid-base equilibria of the incorporated dyes. To this aim, the zeolite was suspended in water and exchanged with an excess of $\mathrm{CsCl}$ under reflux and stirring during $45 \mathrm{~min}$ at $80{ }^{\circ} \mathrm{C}$. Cationic dyes (oxazine 1 and 4 ) were incorporated into $\mathrm{Cs}^{+}$-LTL zeolite by cationic exchange by means of the addition of an aqueous solution of the dye to a zeolite suspension in water at $50{ }^{\circ} \mathrm{C}$ and during $6 \mathrm{~h}$ under stirring and reflux. Neutral dyes (BODIPY, Dmpopop and C165) were incorporated into Cs ${ }^{+}$-exchanged LTL zeolite by using gas phase insertion at high temperatures. After vacuum drying of the zeolite, it was sealed together with the adequate quantity of the dye at vacuum in an ampoule. Then, the dye was sublimated in a rotary oven during 2 days at $170{ }^{\circ} \mathrm{C}$ and $200{ }^{\circ} \mathrm{C}$ for BODIPY and Dmpopop, and C165, respectively. As a last step, the dye-doped zeolite was rinsed with butanol several times to remove the molecules adsorbed onto the external surface of the crystal. 


\subsection{External Functionalization of LTL Zeolite}

LTL zeolite crystals (100 mg) were suspended in $20 \mathrm{~mL}$ of toluene in a previously-silanized glass flask to render the surface inert and avoid any possible reactions between the stopcock molecule and the glass. The number of channel entrances in one crystal was calculated (twice the number of pores, which are around 267,000 for the herein tested disc-shaped crystals of 1- $\mu \mathrm{m}$ diameter) [32], and one equivalent of BODIPY-stopcock molecule was added to the suspension. In the next step, the solution was sonicated for $15 \mathrm{~min}$ to enable the adsorption of the stopcock on the LTL zeolite surface and then heated at reflux for $15 \mathrm{~h}$ (Figure 6). Finally, the crystals were centrifuged and washed twice with methanol to remove the excess of stopcock molecules adsorbed on the surface.

\subsection{Dye Loading Level}

The amount of dye incorporated in the LTL zeolite channels for a certain loading was estimated using the theoretically-calculated (semiempirical AM1) molecular dimensions of the molecule and the unit cell parameters provided by X-ray diffraction [32]. Considering the length of the long axis of the molecule, the adsorption site filled for each dye can be estimated (i.e., the number of unit cells occupied by a dye molecule), being two unit cells for carbostyril, BODIPY and oxazine, whereas three for oxazole. Thus, knowing the number of unit cells in the zeolite sample, the target dye loading is calculated according to the number of adsorption sites desired to be occupied.

\subsection{Spectroscopic and Microscopic Characterization}

The absorption spectra in the solid state (powder) were registered in a Varian spectrophotometer (Model Cary 4E, Agilent Technologies, Santa Clara, CA, USA) by an integrating sphere in which the reflectance is detected instead of the transmittance. The fluorescence steady-state spectra were recorded in the solid state using the front-face configuration in an spectrofluorometer (Model FLSP 920, Edinburgh Instruments, Livingston, UK) and corrected from the monochromators' wavelength dependence, the lamp profile and the photomultiplier sensitivity. Fluorescence images of dye-doped zeolites were recorded with an optical inverted microscope with epi configuration (Olympus BX51) equipped with a color CCD (DP72). Samples excitation was set by a Chroma band-pass filter (350/50), and emission was collected with a Chroma cutoff filter (E400LPv2 from $400 \mathrm{~nm}$ ). The corresponding fluorescence spectra were recorded by a fiber coupling from Olympus microscope to the aforementioned spectrofluorometer.

\section{Conclusions}

The strategy of allocating suitable dyes into the pores of LTL zeolite or plugging the entrances of LTL zeolite with fluorophores has been proven as a successful approach to achieve highly organized bioinspired antenna materials guided by FRET processes, in which dyes are protected and aligned in a preferential disposition avoiding fluorescent-quencher aggregates even at high loadings. Such a cage-protecting effect leads to a highly photostable and reliable material. Indeed, no sign of degradation was detected, neither after long irradiation periods (even with strong light sources as diode lasers), nor with the aging-time (up to several months). These photoactive hybrid materials are able to harvest light from a wide range of the electromagnetic spectrum (UV-Vis) and convert it into red light. Moreover, the overall outcoming fluorescence from the hybrid material can be triggered by just choosing the right dye combination and amount. Parameters such as the donor/acceptor spectral overlap and ratio, ruling the FRET efficiency, and the brightness of the final emitting acceptor, play a key role in this regard. A fine control of such features gives rise to very versatile photoactive materials where not only predominant red emission can be obtained, but also white-light emission (simultaneous blue, green and red emission). Accordingly, the combination of ameliorated FRET efficiency and acceptors with improved fluorescence efficiencies is required to push the antenna effect and consequently achieve dominating red emission, whereas lowering the acceptor 
content (to decrease the FRET efficiency for a given dye mixture) or choosing a final energy trap with lower emission ability is mandatory to attain white-light-emitting materials, where the light can be modulated over a broad spectral range.

The functionalization of the channels entrances with closure molecules based on BODIPY dyes endows further versatility to the material. Indeed, the herein reported stopcock has free chromophoric positions amenable to post-functionalization, rendering multifunctional and smart nanomaterials. As a matter of fact, the methyls groups at the $\alpha$-pyrrolic positions can undergo Knoevenagel condensations, which allow the attachment of aromatic frameworks to the dipyrrin backbone and push the spectral bands deeper into the red part of the visible spectrum or eventually reaching the NIR region. Alternatively, the fluorine atoms of the boron bridge can be replaced through nucleophilic substitutions. In this way, suitable functionalized chains can be grafted at this boron center, without altering the photophysics of the dye, to promote the coupling of the dye-doped material with the target device (i.e., semiconductor for photovoltaics purposes) or to link zeolites to each other. Current work is in progress to test these avenues.

Supplementary Materials: The following are available online at www.mdpi.com/1996-1944/10/5/495/s1: Figure S1 to S3: Spectral overlaps between energy donor and acceptor enabling FRET in each tested dye combination.

Acknowledgments: This work was supported by the Basque Government (IT912-16) and Spanish MICINN (MAT2014-51937-C3-3-P) projects. Leire Gartzia also thanks the Basque Goberment for a Post-doctoral grant. Yi Xiao from Dalian University (China) is kindly acknowledged for the supply and synthesis of the stopcock molecule.

Author Contributions: The research work was fully accomplished and partially written by Leire Gartzia-Rivero assisted in the laboratory work, with the data evaluation and writing manuscript. Iñigo López-Arbeloa supervised the research work, as well as revised the manuscript.

Conflicts of Interest: The authors declare no conflict of interest.

\section{References}

1. Benniston, A.C.; Harriman, A. Artificial photosynthesis. Mater. Today 2008, 11, 26-34. [CrossRef]

2. El-Khouly, M.; El-Mohsnawy, E.; Fukuzumi, S. Solar energy conversion: From natural to artificial photosynthesis. J. Photochem. Photobiol. C Photochem. Rev. 2017, 31, 36-83. [CrossRef]

3. Zan, G.; Wu, Q. Biomimetic and bioinspired synthesis of nanomaterials/nanostructures. Adv. Mater. 2016, 28, 2099-2147. [CrossRef]

4. Scholes, G.D.; Mirkovic, T.; Turner, D.B.; Fassioli, F.; Buchleitner, A. Solar light harvesting by energy transfer: From ecology to coherence. Energy Environ. Sci. 2012, 5, 9374-9393. [CrossRef]

5. Scholes, G.D.; Fleming, G.R.; Olaya-Castro, A.; van Grondelle, R. Lessons from nature about solar light harvesting. Nat. Chem. 2011, 3, 763-774. [CrossRef] [PubMed]

6. Hambourger, M.; Moore, G.F.; Kramer, D.M.; Gust, D.; Moore, A.L.; Moore, T.A. Biology and technology for photochemical fuel production. Chem. Soc. Rev. 2009, 38, 25-35. [CrossRef] [PubMed]

7. Del Guerzo, A.; Olive, A.G.L.; Reichwagen, J.; Hopf, H.; Desvergne, J.P. Energy transfer in self-assembled $[n]$-acene fibers involving $\geq 100$ donors per acceptor. J. Am. Chem. Soc. 2005, 127, 17984-17985. [CrossRef] [PubMed]

8. Nakamura, Y.; Aratani, N.; Osuka, A. Cyclic porphyrin arrays as artificial photosynthetic antenna: Synthesis and excitation energy transfer. Chem. Soc. Rev. 2007, 36, 831-845. [CrossRef] [PubMed]

9. Puntoriero, F.; Sartorel, A.; Orlandi, M.; La Ganga, G.; Serroni, S.; Bonchio, M.; Scandola, F.; Campagna, S. Photoinduced water oxidation using dendrimeric Ru(II) complexes as photosensitizers. Coord. Chem. Rev. 2011, 255, 2594-2601. [CrossRef]

10. Dutta, P.K.; Varghese, R.; Nangreave, J.; Lin, S.; Yan, H.; Liu, Y. DNA-directed artificial light-harvesting antenna. J. Am. Chem. Soc. 2011, 133, 11985-11993. [CrossRef] [PubMed]

11. Fleming, C.N.; Jang, P.; Meyer, T.J.; Papanikolas, J.M. Energy migration dynamics in a Ru(II)- and Os(II)-based antenna polymer embedded in a disordered, rigid medium. J. Phys. Chem. B 2004, 108, 2205-2209. [CrossRef] 
12. Martínez-Martínez, V.; García, R.; Gómez-Hortigüela, L.; Sola Llano, R.; Pérez-Pariente, J.; López-Arbeloa, I. Highly luminescent and optically switchable hybrid material by one-pot encapsulation of dyes into MgAPO-11 unidirectional nanopores. ACS Photonics 2014, 1, 205-211.

13. Lakowicz, J.R. Principles of Fluorescence Spectroscopy, 3rd ed.; Springer: Singapore, 2006.

14. Hötzer, B.; Medintz, I.L.; Hildebrandt, N. Fluorescence in nanobiotechnology: Sophisticated fluorophores for novel applications. Small 2012, 8, 2297-2326. [CrossRef] [PubMed]

15. Claassens, N.J.; Volpers, M.; Santos, V.; van der Oost, J.; de Vos, W.M. Potential of proton-pumping rhodopsins: Engineering photosystems into microorganisms. Trends Biotechnol. 2013, 31, 633-642. [CrossRef] [PubMed]

16. Feng, X.; Ding, X.; Chen, L.; Wu, Y.; Liu, L.; Addicoat, M.; Irle, S.; Dong, Y.; Jiang, D. Two-dimensional artificial light-harvesting antennae with predesigned high-order structure and robust photosensitising activity. Sci. Rep. 2016, 6, 32944. [CrossRef] [PubMed]

17. Ramamurthy, V. Controlling photochemical reactions via confinement: Zeolites. J. Photochem. Photobiol. C Photochem. Rev. 2000, 1, 145-466. [CrossRef]

18. Tao, Y.; Kanoh, H.; Abrams, L.; Kaneko, K. Mesopore-modified zeolites: Preparations, characterization and applications. Chem. Rev. 2006, 106, 896-910. [CrossRef] [PubMed]

19. Brackmann, U. Laser Dyes, 3rd ed.; Lambda Physik AG: Göttingen, Germany, 2000.

20. Schulz-Ekloff, G.; Wöhrle, D.; Van Duffel, B.; Schoonheydt, R.A. Chromphores in porous silicas and minerals: Preparation and optical properties. Microporous Mesoporous Mater. 2002, 51, 91-138. [CrossRef]

21. Calzaferri, G. Nanochannels: Hosts for the supramolecular organization of molecules and complexes. Langmuir 2012, 28, 6216-6231. [CrossRef] [PubMed]

22. Sola-Llano, R.; Martínez-Martínez, V.; Fujita, Y.; Gómez-Hortigüela, L.; Alfayate, A.; Uji-i, H.; Fron, E.; Pérez-Pariente, J.; López-Arbeloa, I. Formation of a nonlinear optical host-guest hybrid material by tight confinement of LDS 722 into aluminophosphate 1D nanochannels. Chem. Eur. J. 2016, 22, 15700-15711. [CrossRef] [PubMed]

23. Corma, A.; Díaz, U.; García, T.; Sastre, G.; Velty, A. Multifunctional hybrid organic-inorganic catalytic materials with a hierarchical system of well-defined micro- and mesopores. J. Am.Chem. Soc. 2010, 132, 15011-15021. [CrossRef] [PubMed]

24. Larlus, O.; Valtchev, V.P. Crystal morphology control of LTL-type zeolite crystals. Chem. Mater. 2004, 16, 3381-3389. [CrossRef]

25. Tompsett, G.A.; Conner, W.C.; Yngvesson, K.S. Microwave synthesis of nanoporous materials. ChemPhysChem 2006, 7, 296-319. [CrossRef] [PubMed]

26. Itani, L.; Bozhilov, K.N.; Clet, G.; Delmotte, L.; Valtchev, V. Factors that control zeolite L crystal size. Chem. Eur. J. 2011, 17, 2199-2210. [CrossRef] [PubMed]

27. Lupulescu, A.I.; Kumar, M.; Rimer, J.D. A facile strategy to design zeolite L crystals with tunable morphology and surface architecture. J. Am. Chem. Soc. 2013, 135, 6608-6617. [CrossRef] [PubMed]

28. Kumar, M.; Li, R.; Rimer, J.D. Assembly and evolution of amorphous precursors in zeolite L crystallization. Chem. Mater. 2016, 28, 1714-1727. [CrossRef]

29. Devaux, A.; Calzaferri, G.; Bleser, P.; Cao, P.; Brüwhiler, D.; Kunsmann, A. Efficient and robust host-guest antenna composite for light harvesting. Chem. Mater. 2014, 26, 6878-6885. [CrossRef]

30. Tabachi, G.; Calzaferri, G.; Fois, E. One-dimensional self-assembly of perylene-diimide dyes by unidirectional transit of zeolite channel openings. Chem. Commun. 2016, 52, 11195-11198. [CrossRef] [PubMed]

31. Insuwan, W.; Rangsriwatananon, K.; Meeprasert, J.; Namuangruk, S.; Surakhot, Y.; Kungwan, N.; Jungsuttiwong, S. Combined experimental and theoretical investigation on fluorescence resonance energu transfer of dye loaded on LTL zeolite. Microporous Mesoporous Mater. 2017, 241, 372-382. [CrossRef]

32. Gartzia-Rivero, L.; Bañuelos-Prieto, J.; Martínez-Martínez, V.; López-Arbeloa, I. Versatile photoactive materials based on zeolite L doped with laser dyes. ChemPlusChem 2012, 77, 61-70. [CrossRef]

33. Vohra, V.; Calzaferri, G.; Destri, S.; Pasini, M.; Porzio, W.; Botta, C. Towards white light emission through efficient two-step energy transfer in hybrid nanofibers. ACS Nano 2010, 4, 1409-1416. [CrossRef] [PubMed]

34. Giasante, C.; Schäfer, C.; Raffy, G.; Del Guerzo, A. Exploiting direct and cascade energy transfer for color-tunable and white-light emission in three-component self-assembled nanofibers. J. Phys. Chem. C 2012, 116, 21706-21716. [CrossRef] 
35. Sens, R.; Drexhäge, K.H. Fluorescence quantum yield of oxazine and carbazine laser dyes. J. Lumin. 1981, 24/25, 709-712. [CrossRef]

36. Gartzia-Rivero, L.; Cerdán, L.; Bañuelos, J.; Enciso, E.; López-Arbeloa, I.; Costela, A.; García-Moreno, I. Förster resonance energy transfer and laser efficiency in colloidal suspensions of dye-doped nanoparticles: Concentration effects. J. Phys. Chem. C 2014, 118, 13107-13117. [CrossRef]

37. Brühwiler, D.; Calzaferri, G. Selective functionalizaion of the external surface of zeolite L. C. R. Chim. 2005, 8, 391-398. [CrossRef]

38. Tabachi, G.; Fois, E.; Calzaferri, G. Structure of nanochannel entrances in stopcock-functionalized zeolite L composites. Angew. Chem. Int. Ed. 2015, 127, 11264-11268. [CrossRef]

39. Lu, H.; Mack, J.; Yang, Y.; Shen, Z. Structural modification strategies for the rational design of red/NIR region BODIPYs. Chem. Soc. Rev. 2014, 43, 4778-4823. [CrossRef] [PubMed]

40. Loudet, A.; Burgess, K. BODIPY dyes and their derivatives: Syntheses and spectroscopic properties. Chem. Rev. 2007, 107, 4891-4932. [CrossRef] [PubMed]

41. Boens, N.; Verbelen, B.; Dehaen, W. Postfunctionalization of the BODIPY core: Synthesis and spectroscopy. Eur. J. Org. Chem. 2015, 6577-6595. [CrossRef]

42. Bañuelos, J. BODIPY dye, the most versatile fluorophore ever? Chem. Rec. 2016, 16, 335-348. [CrossRef] [PubMed]

43. Gartzia-Rivero, L.; Baluelos, J.; Izquierdo, U.; Barrio, V.L.; Bizkarra, K.; Cambra, J.F.; López-Arbeloa, I. Microwave Synthesis of LTL Zeolites with Tunable Size and Morphology: An Optimal Support for Metal-Catalyzed Hydrogen Production from Biogas Reforming Processes. Part. Part. Syst. Charact. 2014, 31, 110-120. [CrossRef]

(C) 2017 by the authors. Licensee MDPI, Basel, Switzerland. This article is an open access article distributed under the terms and conditions of the Creative Commons Attribution (CC BY) license (http:/ / creativecommons.org/licenses/by/4.0/). 